\title{
Dispersão da Urbanização no Território Perspectivas e desafios
}

\section{Francesco Indovina*}

Resumo: Propãe-se uma distinção entre a cidade física, sua morfologia, e a condição urbana, ou seja a vida que se desenvolve na cidade. Costuma-se considerar estes dois aspectos como inseparáveis, afinal é a história da cidade que nos apresenta este vínculo, mas a evolução dos processos de um novo assentamento sugere que a condição urbana se realiza também fora da cidade compacta. Uma confirmação da "necessidade" da cidade para a espécie humana. Este fenómeno pode conduzir, se for gerido com visão de longo alcance, à realização da metrópole territorial. Também neste caso, trata-se de uma condição metropolitana fora dos modos tradicionais de organização da metrópole, que torna acessível a condição metropolitana a uma população extensa sem os elementos negativos da grande aglomeração.

Palavras-chave: Aglomeração; Dispersão; Densificação; Condição urbana; Explosão da cidade; Metrópole territorial.

Vorrei iniziare questa comunicazione con un punto controverso che proverei a enunciare in questo modo: la morfologia urbana cambia mentre la condizione urbana persiste.

È evidente che in quest'affermazione provo a scindere l'aspetto morfologico della città, la sua struttura fisica, la sua organizzazione spaziale, dalla sua condizione sociale, da quella che è possibile chiamare "vita urbana".

Si fa fatica a scindere quello che ci appare come inscindibile; siamo soliti guardare alla città nella sua interezza ed essa ci appare come un insieme inscindibile di organizzazione spaziale e sociale. Tutte le esplorazioni della città, da quelle proprie dell'urbanistica a quelle geografiche a quelle letterarie, antropologiche e sociologiche ci parlano prevalentemente di questa unitarietà.

Del resto se osservassimo la storia millenaria del processo di inurbamento della specie umana, dovremmo convenire che la crescita fisica della città e la cultura urbana ci appaiono strettamente collegate; non esiste l'una senza l'altra.

Eppure è possibile ipotizzare che tale visione della città costituisca una distorsione, o forse una semplificazione. Secondo questo punto di vista quello che prevale è l'aspetto morfologico per cui è città quella forma dell'insediamento umano che si presenti come densa, intensa e senza soluzioni di continuità (qualcuno potrebbe anche azzardare, in forma metaforica, quella "dentro le mura").

Non voglio negare che nella storia dell'inurbamento questi connotati sono stati sicuramente non solo presenti ma determinanti. Tuttavia voglio sottolineare che abbiamo continuato a nominare con lo stesso nome "oggetti" molto diversi tra di loro, intendo diversi nel profondo. Non voglio allargare il punto di vista fino a comprendere realtà dell'urbanizzazione molto diverse da quelle che possiamo chiamare "il modo europeo moderno di essere dell'urbano", ma all'interno di questo mondo abbiamo continuato ad usare lo stesso termine "città" per situazioni forse molto diverse tra di loro.

Quale è il dato unificante tra una città del 1600 con quello di una città industriale del 1800? Niente della vita urbana e dell'organizzazione del funzionamento stesso della città si presenta uguale, ad eccezione, appunto, dell'intensità, densità e assenza di soluzione di continuità. Ma le condizioni di vita urbana in queste due città, lontane un paio di secoli, non hanno niente in comune. $\mathrm{E}$ ancora quale

\footnotetext{
*Università IUAV di Venezia, Facoltà di Architettura di Alghero. Direttore della collana 'Studi Urbani e Regionali' della Franco Angeli, co-fondatore della rivisla Archivio di Studi Urbani e Regionali (ASUR).
} 
differenza introduce nella vita urbana l'espansione del welfare state? Eppure si è continuato ad usare lo stesso termine guardando più la situazione morfologica piuttosto che quella sociale e di vita urbana.

A me pare che se si volesse indagare la condizione urbana di questa fase storica sarebbe opportuno scindere i due aspetti, non perché uno sia più importante dell'altro, ma soltanto perché i due non risultano più strettamente collegati. La condizione urbana, cioè, non necessità per il suo concreto manifestarsi di una specifica condizione fisicomorfologica caratterizzata da densità, intensità e assenza di soluzione di continuità. $\mathrm{O}$ detto in altro modo, forse più provocatorio, la dispersione non nega la condizione urbana.

Si avanza l'ipotesi che la condizione urbana non è (solo) agglomerazione, non è (solo) densità e intensità edilizia, non è (solo) compattezza, ma i suoi dati caratterizzanti sono da ricercarsi in ambito "sociale" e nelle condizioni di vita. In particolare, e solo in modo esemplificativo, si possono indicare i seguenti caratteri come quelli che permettono di identificare tale condizione:

- l'ampiezza delle relazioni sociali, e si possono mettere tra queste quelle che hanno natura intensa, sia anche quelle che è possibile definire "superficiali", gli uni e gli altri rilevanti per la colloquialità urbana che costituisce la modalità attraverso la quale si manifesta l'ampliamento delle relazioni sociali;

- la cultura urbana, vista sotto diversi aspetti. Come espressione, spesso anche caotica, di somma di esperienze codificabili anche in comportamenti non omogenei ma che arricchiscono lo sguardo sul mondo. Si tratta, ancora, dell'ambiente specifico adatto alla crescita e sperimentazione di forme artistiche. Per non parlare delle condizioni, anche in termini di organizzazione, per lo sviluppo della scienza e del sapere;

- la dotazione dei servizi collettivi, aspetto questo che non pare abbia bisogno di ulteriore specificazioni e che da sempre distingue la città dalla non città;

- la partecipazione politica, intendendo non solo l'espressione di voto, ma piuttosto l'interessamento, individuale, di gruppi o collettivo alle scelte di governo. Non solo nelle forme in qualche modo istituzionalizzate, ma anche di quelle spontanee (il massimo di "partecipazione", si ha con il conflitto);
- il "risarcimento sociale", si può sostenere che la condizione urbana in quanto tale costituisce, per tutto quello detto prima, una forma in qualche modo di "risarcimento" sociale per individui e strati sociali più penalizzati dal meccanismo economico sociale. Non ci si riferisce tanto alla possibilità che negli interstizi urbani questi possano trovare qualche soluzione ai loro problemi, ma piuttosto al fatto che la condizione urbana, pur non risolvendo la loro situazione, li inserisce in un contesto di vitalità, di organizzazione, di cultura che in qualche modo tende a rompere il loro isolamento;

La condizione urbana è ancora caratterizzata da accoglienza, da diversità e da imprevedibilità, tutte condizioni di vitalità. Sarebbe possibile identificare altri caratteri specifici della condizione urbana, tuttavia i precedenti paiono sufficienti se accompagnati da due altre osservazioni.

La prima vuole essere un richiamo al realismo, non si faccia l'errore di idealizzare la città del passato. La condizione urbana ha le caratteristiche indicate ma queste si accompagnano a fenomeni negativi (conflitti, contraddizioni, emarginazione, degrado, ecc.). Per fare solo un esempio "accoglienza" e "razzismo" sono due modalità che si riferiscono allo stesso fenomeno, l'immigrazione, ma mentre il primo termine si pone positivamente (che non vuol dire banalmente "buonista", ma piuttosto attento alle modalità attraverso le quali garantire all'immigrato l'accoglienza, sapendo che si va incontro ad una convivenza spesso "difficile"), il secondo si caratterizza per il rifiuto. Ma attenzione l'uno o l'altro atteggiamento caratterizzano il clima culturale, sociale e anche politico della città a determinare il quale ha rilievo e influenza la qualità del governo.

Spesso di fronte alle trasformazioni della città, si fa appello, indirettamente, ad una "età del l'oro" della città del passato, dove tutte le caratteristiche negative scompaiono, una situazione mai esistita; la città per sua natura è luogo di contraddizioni e di conflitti, di perturbazioni, ma anche di avanzamenti culturali e sociali; è il "governo" della città che determina la qualità della città (una città è "bella" solo se "buona", se cioè è caratterizzata da "buon governo").

La seconda osservazione vuole sottolineare che molti (tutti?) degli aspetti che caratterizzano la 
condizione urbana, fanno riferimento all'esistenza di strutture, organizzazioni, enti, ecc. preposti alla gestione o alla facilitazione, o all'attivazione di quella che chiamiamo condizione urbana (gli spazi pubblici, le scuole, gli ospedali, le biblioteche, i teatri, i centri di ricerca, ecc.). Questo per sottolineare una relazione stretta tra la condizione urbana e la dotazione di "capitale" della città.

Come spero si sia compreso bisogna considerare la "dimensione" una variabile qualitativamente positiva della condizione urbana. La crescita della città, fino alla metropoli, determina, in generale e nell'esperienza europea, un miglioramento della condizione urbana. Questo perché la dimensione accresce il capitale fisso sociale della città, aumentando la possibile di attivare servizi (pubblici e privati) più avanzati e più sofisticati; la liberta individuale aumenta in relazione al diminuire del "controllo sociale"; la moltiplicazione delle opportunità economiche non solo migliora la condizione media ma promuove, anche se limitatamente, la mobilità sociale; si moltiplicano, proprio in ragione dell'aumentato numero di potenziali utenti, l'offerta e le occasioni culturali. La mobilità delle persone presenta nel passaggio dalla piccola alla grande dimensione una variazione importante e imponente: nella piccola comunità la mobilità interna è di tipo pedonale, con il crescere della comunità questa diminuisce drasticamente e aumenta in modo esponenziale quella meccanica individuale che, ad un certo punto, viene contrastata dai mezzi collettivi. Quella della mobilità può essere assunta come un esempio indicativo dei cambiamenti che intervengono con la crescita dimensionale della città fino alla metropoli.

Ma le medaglie, come è noto, hanno due facce, così alla faccia degli esiti positivi determinati dalla crescita si contrappone la faccia degli effetti negativi sulla qualità urbana. Effetti non meno importanti dei primi. Intanto tende a crescere la specializzazione sociale dello spazio, fino, nei casi estremi, ad avere una città o metropoli fatta di zone tra di loro poco permeabili, ciascuna chiusa in una propria identità (un proprio egoismo); nonostante i servizi crescono i casi di "povertà urbana" e di isolamento, persone non in grado di utilizzare le opportunità che la città offre; cresce a dismisura la congestione, nonostante il contrasto portato alla mobilità individuale da quella collettiva, e produce effetti negativi non solo sulla qualità della vita dei singoli ma anche sui costi di produzione delle attività produttive e dei servizi. L'aumento di capitale sociale fa incrementare la rendita e con questo il "costo della città", fenomeno che produce l'espulsione di famiglie e di attività economiche dalla città, perché non in grado di sostenere i maggiori costi. Cresce il senso di insicurezza, mentre nella piccola comunità tutti sono "familiari" nella metropoli tutti sono "estranei", l'insicurezza può essere considerata un costrutto sociale politicamente utilizzato, un utilizzo politico che si avvantaggia dell'effetto di estraneità che può produrre la dimensione.

Può essere opportuno guardare al problema della crescita urbana, di cui si è cercato di illustrare vantaggi e svantaggi, dal punto di vista del suo motore. Possiamo considerare l'agglomerazione come il motore di tale crescita fino alla metropoli. L'agglomerazione, il concentrarsi nello spazio di uomini e capitale, determina dei vantaggi e delle convenienze, reali o immaginari, non importa. Vantaggi per le famiglie e per gli individui, vantaggi per le imprese e le attività economiche, in un processo cumulativo e circolare: maggiore dimensione più vantaggi, più vantaggi più attrazione, più attrazione maggiore dimensione.

È propria questa la "causa" che è possibile individuare come motore del processo di concentrazione di capitale e popolazione. Tutte le ipotesi antiurbane non hanno avuto il successo sperato, nonostante le "meraviglie" che prospettavano, proprio per non aver considerato la potenza e la forza dell'agglomerazione.

L'agglomerazione, determinando la crescita dimensionale della città, produce, tuttavia, gli aspetti negativi di cui si è detto in precedenza. È possibile tradurre questo fenomeno mettendo in chiaro che i vantaggi dell'agglomerazione arrivati ad un certo punto di dimensione urbana (e di mancanza di governo adeguato) si trasformano in svantaggi. Ovviamente non si tratta né di un processo istantaneo, né tanto meno sono individuabili delle soglie standard dimensionali raggiunte le quali i vantaggi si trasformano in svantaggi. Condizioni specifiche di luogo, di sviluppo e, soprattutto, di governo determinano soglie diverse di questo passaggio. Quello che, tuttavia, va rilevato è il deteriorarsi del processo di agglomerazione agli occhi degli abitanti ed ai conti delle attività economiche.

In sostanza la forza dell'agglomerazione si indebolisce, un fatto questo che determina quel feno- 
meno che è stato chiamato di esplosione urbana, di metropolizzazione del territorio, della costruzione di una città di città, della costruzione dell'arcipelago metropolitano o della metropoli territoriali. Nomi diversi per definire un fenomeno molto simile che si presenta in molti parti del continente europeo, le cui peculiarità di luogo non mettono in discussione l'unitarietà del fenomeno.

Prima di dare una sintetica descrizione del fenomeno dell'esplosione urbane può essere utile esaminare le forze generatrici di tale processo.

Si è già individuato nell'indebolimento della forza dell'agglomerazione una di questi fattori, a questo, tuttavia, se ne possono sommare altri che hanno dato un senso specifico al fenomeno dell'esplosione urbana e ne hanno determinato i contorni e le linee di tendenza.

Piace segnalare:

- l'innovazione tecnologica è sempre presente in ogni trasformazione della città, ma si rifletta che mentre nel passato l'innovazione riguardava soprattutto i modi organizzativi e di funzionamento della città (dai mezzi di trasporto collettivo, alle reti urbane, all'illuminazione, ecc.); oggi l'innovazione, nonostante sia fortemente inclusiva, segna uno scarto: l'innovazione usata dalle famiglie e dalle imprese è superiore a quella utilizzata nella e dalla città. Fenomeno questo che, in un certo senso, spiega perché molto spesso gli individui non sopportano la città;

- dove l'innovazione ha avuto una grande portata è stata nel processo produttivo, comunque inteso. Per quello che riguarda il tema qui considerato si osservino i seguenti effetti: il minore consumo di spazio necessario per uno stesso processo produttivo, che rende spesso vantaggiosa una rilocalizzazione; economie di scala raggiungibili non con la concentrazione della produzione in un unico luogo ma con la gestione (tecnologicamente avanzata) di unità produttive di piccola dimensione e diffuse nel territorio (anche in paesi diversi); i vantaggi di "vicinato" con altre imprese raggiungibili anche a distanza; l'esternalizzazione di parti del processo produttivo o di funzioni aziendali che smagriscono le imprese; la capacità di controllo della catena di produzione del valore aggiunto determina fondamentali guadagni alle imprese. Questi fenomeni, ed altri ancora, svincolano l'impresa dalla città e rendono effettive localizzazioni alternative ampie;
- sono evidenti, senza necessità di spiegazioni, come siano mutati gli stili di vita di quote rilevanti di popolazione. Quello che si vorrebbe sottolineare come un aspetto fondamentale delle questioni qui affrontate è la crescita della mobilità individuale (con mezzi diversi, ma con l'auto soprattutto), cosa che ha ridotto l'importanza della "distanza" e quindi delle localizzazioni "vicine";

- il "successo" della città, la sua sempre più ampia dotazione di capitale fisso sociale, ha determinato la crescita della rendita e per conseguenza il costo della città, generando espulsione di famiglie e imprese non in grado di sopportare tali maggiori costi e alla ricerca di luoghi di insediamento più convenienti;

- una forte componente culturale (ideologica) ha spinto sempre più famiglie alla ricerca di stili dell'abitare non di tipo urbano: casa singola, casa con giardino, casa isolata, presunta aria di buona qualità, ambiente verde, ecc.

L'insieme di questi fenomeni, accavallandosi uno sull'altro, in tempi diversi e con esiti non sempre omogenei ha determinato il fenomeno dell'esplosione urbana, che è possibile sinteticamente indicare come la localizzazione di "oggetti" e funzioni di tipo specificatamente urbano, nel territorio ampio, si potrebbe dire fuori le mura. Ci si riferisce ad abitazioni, fabbriche, servizi commerciali, servizi per il tempo libero, insediamenti di edilizia economica e popolare, ecc. Quello che si nota osservando questi territori è, appunto, una città esplosa, dilatata nel territorio e che ha perso la sua compattezza.

Se si volesse tracciare il processo dell'esplosione urbana, o meglio le fasi attraverso le quali si giunge a quella che proponiamo di chiamare o arcipelago metropolitano o metropoli territoriale (o dare al fenomeno gli altri nomi prima indicati), si dovrebbero mettere in luce i seguenti passaggi:

- urbanizzazione della campagna: che costituisce la prima fase nella quale nella "campagna" vanno a localizzarsi le prime strutture (abitazioni, imprese, ecc.) che non hanno attinenza con l'attività agricola;

- urbanizzazione diffusa: che costituisce un ampliamento quantitativo della fase precedente, ma non una modifica di senso; la campagna resta ancora il luogo dell'agricoltura e gli insediamenti non legati all'agricoltura, ancorché numerosi, appaiono ancora come una sorta di anomalia; 
- città diffusa: in questo caso si colloca un passaggio di senso. Non solo quantitativamente gli insediamenti non agricoli diventano sempre più estesi, ma si localizzano nel territorio extra-urbano servizi di qualità urbana (centri commerciali, ipermercati, attrezzature per lo sport e il tempo libero, multisala cinematografica, centri di divertimento, ecc.). In questo contesto il territorio ampio dell'insediamento viene usato come una città (appunto città diffusa), una città dilatata è priva dei connotati morfologici specifici (intensità, densità e assenza di soluzioni di continuità). Un fenomeno questo al quale attribuiamo un significato molto importante: la continua ricerca di una condizione urbana anche in assenza dei caratteri morfologici specifici della tradizione;

- metropoli territoriale: si è di fronte ad un ulteriore passaggio. Nei territori della città diffusa, le città di maggior dimensione hanno continuato a conservare al loro interno i centri di governo (in senso ampio) e i centri di eccellenza. In questa situazione la città diffusa è ancora caratterizzata da gerarchie territoriali anche se attenuate. Nella fase successiva questi centri di governo (politici, amministrativi, finanziari, dell'informazione, ecc.) e i poli di eccellenza (della ricerca, della sanità, ecc.) abbandonano le città più grandi e si vanno a localizzare in centri di minore dimensione o ne sorgono di nuovi scegliendo localizzazioni che non sono quelle canoniche delle città maggiori. Questo fenomeno innalza complessivamente la qualità di questo territorio facendole assumere il ruolo di una metropoli, sia dal punto di vista della popolazione che è molto più numerosa, sia dal punto di vista delle attrezzature e dotazioni.

Va precisato, tuttavia, che il processo che qui si propone, in modo molto semplificato e schematico, non solo, nella realtà, risulta più complesso e talvolta caotico, ma soprattutto non deve essere interpretato come la proposizione di una linea evolutiva obbligata: dalla campagna alla metropoli territoriali.

Inoltre le condizioni del luogo e situazioni economiche, sociali e culturali specifiche, possono dare esisti diversi, ancorché assimilati dalla stessa logica. Così come nel passato si sono avute città molte diverse tra loro, ma unite dalla stessa logica di funzionamento, così nel futuro si potranno avere metropoli territoriali molto diverse tra di loro ma con caratteri e logiche di funzionamento simili.
Si sostiene, per le ragioni dette in precedenza, che il processo di diffusione e di metropolizzazione del territorio caratterizza l'epoca attuale e che quindi la città diffusa e la metropoli territoriale costituiscono le forme tendenziali dell'organizzazione del territorio dove si manifesta la condizione urbana.

Quando si dice metropoli territoriali non si fa riferimento ad entità senza, saranno diverse tra di loro; così quella che potrà realizzarsi nel caso in cui preesiste una forte città metropolitana(il caso di Barcellona) non sarà identica a quella che potrà $n$ in un territorio caratterizzato da città medie nessuna in grado di prevalere (come il Veneto centrale).

Pur assumendo come costitutiva questa diversità, le metropoli territoriali hanno (e avranno) dei tratti fortemente comuni. Di questi si vorrà offrire una descrizione semplificata e, in un certo senso, stereotipata da tre diversi punti di vista: la descrizione spaziale, la funzionalità e la società.

Dal punto di vista spaziale avremo un territorio fortemente e interamente interconnesso costituito da città, anche di antico insediamento, di diversa dimensione (fino alla presenza di una tradizionale grande città), a questi si sommano in misura e dimensione diversa nuclei di insediamento diffuso e abitazioni sparse. La rete infrastrutturale sarà molto ampia con la presenza di autostrade, strade, vie di tipo urbane, vie commerciali, strade mercato, ecc. e ai nodi di tale rete, quali punti di massima accessibilità si troveranno poli di servizi, per lo più commerciali. Altre zone di specializzazione funzionale (per il tempo libero, lo sport, il divertimento, ecc.) si potranno individuare sempre in vicinanza dei nodi di accessibilità. Le attività produttive risultano localizzate in zone specificatamente attrezzate a questo scopo, o anche isolate nel territorio.

Questa fotografia si completa con l'esistenza di parchi, giardini, e spazi per attività all'aperto; mentre zone di campagna produttiva costituirà il tessuto connettivo ancorché di fatto marginale sia dal punto di vista economico che nel disegno del paesaggio.

Tra tutto questo "costruito" sarà facile individuare spazi residuali di dimensione non adatte per essere utilizzati o in "attesa" di una loro valorizzazione edilizia.

Dal punto di vista della funzionalità, pur assumendo che non possa esistere un territorio assolutamente privo di gerarchie, i fattori che costruiscono fenomeni gerarchici sono, infatti, molteplici e 
spesso non eliminabili (di tipo storico, naturalistico, ecc.), si nota l'esistenza di una gerarchia territoriale di tipo soft, le gerarchie hard preesistenti si sono depotenziate e non se ne sono realizzate di nuove.

Proprio l'attenuazione del fenomeno gerarchico determina una mobilità delle persone, sia obbligatoria che volontaria multidirezionale. Ogni punto del territorio, infatti, "serve"la popolazione dell'intera metropoli territoriale. I flussi di merci e informazioni saranno anch'essi multidirezionali e crescenti. L'ampliamento delle relazioni che si possono istaurare in questo territorio determina una forte potenzialità alla crescita economica; la diffusione, cioè, non corrisponde né genera depressione economica, ma piuttosto crea potenzialità economiche nuove.

Dal punto di vista della società la metropoli territoriale genera fenomeni di un certo rilievo. Intanto si segnala un'attenuazione dell'organizzazione sociale dello spazio e l'esaltazione della segregazione, con la crescita, anche se ancora in modo limitato, esclusivamente per fasce sociali alte, difese da muri, telecamere, guardie armate ecc. La tendenza prevalente, tuttavia, sembra quella dell'attenuazione della specializzazione sociale degli insediamenti, fenomeno questo dovuto ad un più alto livello di "accoglienza", soprattutto negli insediamenti di minor dimensione (dove più alto è il controllo sociale, maggiore pare la disponibilità ad integrare flussi di immigrazione), ovviamente relazionati alla dimensione dell'insediamento.

La dotazione di servizi metropolitani determina un uso del territorio di tipo metropolitano. Intendiamo con questo termine far riferimento insieme ad un processo di isolamento e di integrazione. In generale la maggior parte delle persone è caratterizzata da una sorta di lacerazione psicologica: si desidererebbe abitare in una piccola comunità caratterizzata da rapporti affettivi, ma la vita in questa stessa comunità appare insopportabile e contrastata dal desiderio della via della grande città; ma contemporaneamente, abitare nella grande città genera il suo rifiuto con la speranza di rifugiarsi in un piccolo centro. Nella situazione della metropoli territoriale è possibile che ciascuno scelga la tipologia dell'abitare preferita senza necessariamente scartare l'altra, proprio in ragione dell'integrazione spaziale e della "necessità" individuale di frequentare (per lavoro, studio, acquisti, amicizie, tempo libero, ecc.) l'intero territorio metropolitano.
A questo punto si ritiene necessario introdurre alcune considerazioni circa i limiti del processo appena descritto. Sebbene sia possibile considerare la nuova configurazione spaziale come "necessaria" (figlia dei cambiamenti che si è cercato di mettere in evidenza) e "opportuna" (per i miglioramenti introdotti nella "condizione urbana"), non si può fare di questa nuova forma di organizzazione del territorio una sorta di mitologia. La metropoli territoriale sarà caratterizzata dalle contraddizioni che la città ha già sperimentato e che sono determinati, in larga parte, dai rapporti sociali di produzione. Per dirla in modo molto sintetica una società iniqua non può avere una città equa, qualsiasi sia la forma di questa. Detto questo, tuttavia, vale la pena di soffermarsi sui limiti specifici della città diffusa $\mathrm{e}$ della metropoli territoriale.

Una quota non piccola dei suoi limiti dipende dal fatto che questo processo, tranne casi rari, non risulta essere frutto di un'azione di governo, ma piuttosto l'esito di un processo di auto-organizzazione. La diffusione, così come si manifesta in molte parti del nostro continente, è il frutto di scelte autonome e individuali di singole famiglie, operatori, imprese, organizzazioni, ecc. (le "pratiche sociali"), scelte che rispondono a soddisfare bisogni o a utilizzare opportunità, e al continuo manifestarsi della crescita, in modo cumulativo, sia delle opportunità che delle necessità. Scelte che si sono avvalse o dall'assenza di ogni regola per la trasformazione del territorio, o dalla ricerca di margini offerti da interpretazioni forzate della legislazione vigente (e qualche volta in disprezzo di tale legislazione), o in relazioni a politiche attivate dai singoli enti territoriali per finalità proprie. Insomma la metropoli territoriale nasce e cresce al riparo di un governo complessivo; gli atti di governo parziali, nel senso che si applicavano ad una parte soltanto nel territorio delle singole amministrazioni comunali, in molti casi possono avere generato un peggioramento della situazione complessiva.

Non si vuole sostenere che un governo complessivo avrebbe potuto eliminare tutte le contraddizioni, ma sicuramente avrebbe forse generato un territorio più funzionalizzato, meglio organizzato e avrebbe esaltato di più le opportunità (per tutti). Ma non inferiori sono i danni prodotti da una cultura passatista, che per anni ha giudicato il fenomeno della dispersione come un elemento di degenerazione e di degradazione della città, senza 
la capacità di vedere non solo che sotto traccia nella diffusione si concretizzava un processo di densificazione metropolitano, ma anche i vantaggi generati da tale fenomeno.

Il risultato della mancanza di un governo della metropoliti territoriale in formazione ha generato un situazione di iper-dotazione di strutture private (soprattutto di tipo commerciale) e di ipo-dotazione di strutture pubbliche, a partire dallo spazio pubblico, dalle infrastrutture in grado di "guidare" l'insediamento, ecc.

Ovviamente un insediamento territoriale non guidato e sotto dimensionato di strutture pubbliche porta ad un alto consumo di suolo, risulta ad alto consumo energetico, sia per le attività domestico che per il trasporto, ad una concorrenza per l'appropriazione dello spazio, ad una sostanzialmente non razionale organizzazione spaziale.

Una metropoli territoriale, proprio per lo specifico carattere "diffuso" presenterà un costo di gestione pubblica (servizi, reti tecnologiche, ecc.) superiore se paragonati al costo di una situazione concentrata; in un situazione di carenza di governo questo fenomeno si accentua, determinando un abbassamento dei servizi. Dove una qualche forma di governo si è manifestata la metropoli territoriale appare più organizzata e con meno consumo di suolo e di energia, e la sua gestione pubblica avviene a costi più contenuti.

Va detto che la gelosa difesa del potere sul territorio, da parete delle amministrazioni locali, in tutti i paesi, rende particolarmente difficile e problematica ogni idea di governo complessivo. Ma se fosse vero che il processo di metropolizzazione si presentasse come una costante la necessità di un governo allo stesso livello imporrebbe modifiche legislative non di poco conto.

Nonostante questi limiti, si possono considerare di grande prospettiva le opportunità che questa nuova struttura territoriale mostra di avere in sé e che ancora non ha completamente manifestato. Sia perché si deve ritenere la metropoli una "forma" territoriale non solo quantitativamente ma anche qualitativamente superiore alla città, sia perché la metropoli territoriale permette l'accesso ad una situazione e cultura metropolitana ad una popolazione ampia riducendo, nello stesso tempo, i disaggi tipici della metropoli tradizionale e qualificando al meglio la stessa vita metropolitana. Inoltre le ampie relazioni possibili al suo interno, svincolate dai costi e dagli ostacoli della congestione, possono creare nuove opportunità di sviluppo economico.

La nuova struttura territoriale se non governata, tuttavia, può generare processi involutivi tali da metterne in crisi le opportunità, senza la possibilità di un ritorno alla situazione anteriore. Il problema del "che fare" pare, a questo punto delle trasformazioni, una domanda urgente e importante ma anche una domanda che non ammette facili risposte non solo per la natura dei problemi ma anche per la vischiosità delle scelte politiche.

Il "che fare", si pensa, dovrà prendere le mosse da tre proposizioni guida e porsi tre obiettivi.

Le tre proposizioni sono:

- l'auto-organizzazione costituisce la realizzazione di nuove situazioni che si riferiscono a nuove necessità e opportunità (tecnologiche, sociali, culturali, di vita, ecc.). Essa si manifesta attraverso pratiche sociali che introducono nel contesto dinamismo e innovazione e mentre producono nuove soluzioni fanno emergere necessità nuove;

- l'auto-organizzazione, e le relative pratiche sociali, presentano dei limiti complessivi per la loro parzialità e individualità, aprono ulteriori contraddizioni, generano situazioni territoriali caratterizzate da sprechi e da irrazionali organizzazioni spaziali;

- la metropoli territoriale non può essere il risultato somma di scelte individuali e di autoorganizzazione, così come non lo è stato la città tradizionale.

Per quanto riguarda gli obiettivi essi possono essere espressi in forma sintetica e apodittica in:

- salvare i dati positivi (innovazione, dinamismo, ecc.) dell'auto-organizzazione e delle pratiche sociali relative che siano coerenti con un disegno di prospettiva;

- eliminare gli elementi negativi delle pratiche sociali e dell'auto-organizzazione affermando un punto di visto e un interesse generale al quale, per quanto possibile, devono conformarsi le pratiche individuali;

- esaltare la metropoli territoriale in tutti i suoi aspetti e opportunità.

La realizzazione di questi obiettivi impone la necessità di affrontare sfide culturali, scientifiche e di governo. A queste saranno dedicate le note di chiusura nella consapevolezza, tuttavia, di non riuscire a mettere in evidenza compiutamente la profondità dei contenuti che tali sfide suggeriscono. 
La sfida "culturale" deve mettere in discussione, come sempre, opinioni consolidate. Bisogna accettare che nel tempo, con le modifiche indotte da trasformazioni economiche, sociali e tecnologiche, si è modificata, fino a mettere in discussione alcune delle sue caratteristiche, la natura della città. E se, come dice Pig Pen, uno della banda dei Peanuts di Charles Shultz, la polvere dei secoli rende tutto omogeneo, nel nostro caso la polvere dei secoli ha fissato una specifica immagine della città che non riusciamo ad abbandonare e che rende opache le trasformazioni. La rapidità dei cambiamenti e il variare dello stesso statuto della città (la città fuori le mura, la metropoli territoriali), impongono una riflessione culturale circa i connotati specifici della città che possono (devono) essere conservati e le modificazioni che devono (possono) essere accettate.

Come più volte ripetuto la condizione urbana deve essere mantenuta, anche se aggiornata, mentre la tradizionale morfologia urbana deve convivere con le nuove morfologie urbane e metropolitane. $\mathrm{Si}$ fa fatica oggi, in molti territori, a riconoscere l'urbano a partire dal segno sul territorio, l'urbanizzazione dilaga nel territorio. Osservando anche solo le cartografie offerte da google in moltissimi casi appare impossibile definire i confini della città.

Non si disconosce il patrimonio (storico, culturale, artistico) consolidato nella città tradizionale, né tanto meno si pensa che questo possa essere abbandonato a deperimento al contrario la sua fruizione nel nuovo contesto può essere più estesa, più intenzionale, più riflessiva perché meno "quotidiana" proprio perché non costituisce più il connotato di riconoscimento della condizione urbana. È questo il lascito più rilevante della storia della città, esso oggi dilaga nel territorio ampio definendo nuovi rapporti interpretativi e di senso.

I patrimoni delle città che costituiscono o costituiranno la metropoli territoriale, non solo andranno valorizzati da una fruizione più ampia ma devono $\mathrm{e}$ possono essere esaltati come valenze significative di appartenenza.

Quando si è detto che la condizione urbana costituisce il lascito più importante della storia della città, non si è, ovviamente, fatto riferimento a qualcosa di stabile, anche la condizione urbana si evolve si adegua ai tempi, agli sviluppi delle cultura e della scienza, alla dinamica economica e sociale, alla tecnologia, ma a qualcosa che pare mantenere i suoi caratteri originari già citati (ampiezza di relazioni sociali, cultura, partecipazione politica, accoglienza, ecc.) anche se trasformati dagli eventi e aggiornati.

È possibile individuare un passaggio di un qualche rilievo nel transito dalla città alla metropoli territoriale. La condizione urbana è una costruzione sociale risultato, insieme, dai condizionamenti dipendenti dall'organizzazione dello spazio fisico, dalle modalità con cui i singoli organizzano la loro vita quotidiana, dalle mode, dai livelli di istruzione e di sapere, dalla tecnologia e di altro ancora. Va riconosciuto che la "concentrazione" ha permesso, in passato, il concretizzarsi della condizione urbana in modo abbastanza facile e si potrebbe dire spontaneo. Ci si deve abituare a considerare che le condizioni contemporanee di insediamento rendono possibile, ancora, la realizzazione di condizioni urbane, anche nel caso della dispersione e della metropoli territoriale. Bisogna prendere atto che questi casi subiscono meno gli effetti dello spazio fisico micro (tipo urbano) e che l'influenza dell'organizzazione spaziale a maglie larghe non condiziona più di tanto la realizzazione della condizione urbana, ma il suo concretizzarsi è meno facile e spontaneo. Un aspetto questo che richiama con forza la necessità, nella costruzione della condizione metropolitana, di apporti culturali in grado di contrapporsi e arricchire la "banalità" del quotidiano. Insomma la metropoli territoriale ha bisogno di un impegno culturale in grado di cucire e ricucire le diverse esperienze, le diverse "culture", i diversi stili di vita, ed ogni altra manifestazione sociale, non tanto per attivare un processo di omogeneizzazione, il che sarebbe stupido, riduttivo e sostanzialmente impossibile, ma per dare forma articolata ad una condizione "urbana metropolitana" aperta, ricca e densa, dove ciascuno possa riconoscersi pur nelle differenze (che sono sempre più accentuate).

Le sfide scientifiche alle quali ci richiama la metropoli territoriale appartengono a diversi orizzonti. $\mathrm{Al}$ primo punto, si potrebbe sostenere, appare indispensabile l'elaborazione di un nuovo concetto di metropoli (appunto la metropoli territoriale, o come altro si vorrà chiamare) che corrisponda ai fenomeni come si stanno manifestando in relazioni alle necessità e opportunità che si sono materializzate.

A partire da questa rinnovata modalità insediativa meritano di essere elaborati strumenti e metodi scientifici in grado di affrontare le questioni più im- 
pellenti che la nuova situazione pone. La soluzioni di queste questioni ha, ovviamente, un correlato di "governo", di cui si dirà più avanti, ma un sapere rinnovato e strumenti scientifici adatti appaiono indispensabili per ben governare; anche se è noto che per ben governare gli strumenti scientifici sono indispensabili ma non sufficienti.

Di seguito si indicheranno alcuni temi che dovrebbero essere affrontati per qualificare in modo positivo la metropoli territoriale e anche per costruire quel nuovo concetto di metropoli di cui si è detto.

Gli obiettivi (di ricerca) che potrebbe essere di grande utilità affrontare, in modo sintetico, paiono:

- per quanto riguarda l'ambiente, uscire dalle ripetizione per porsi certo la questione della salvaguardia, ma anche una elaborazione che veda l'ambiente, qualsiasi cosa questo significhi, un contributo di valorizzazione e di qualificazione dell'insediamento. Mi pare che si possa dire che mentre ampia è la strumentazione scientifica analitica povera, se non di buon senso nei migliori dei casi, quella riferita all'operatività. L'idea di un uso parsimonioso delle risorse pare convincente, ma esso va mediato con le nuove esigenze;

- strumenti in grado di suggerire una razionale moltiplicazione della mobilità (di tutti). Se da una parte è ovvio il necessario spostamento, per quanto possibile, dalla mobilità individuale a quella collettiva, la condizione di dispersione pone problemi nuovi, che forse possono essere adeguatamente affrontati (e in qualche caso già ci si sta provando) anche facendo riferimento alle nuove tecnologie. Pare utile (indispensabile?) una nuova elaborazione scientifica del problema;

- la concezione tradizionale di zonizzazione spaziale, non pare adeguata alla nuova dimensione. Certo va privilegiato il mix sociale e funzionale, vanno individuate le incompatibilità, ma non pare che la nuova dimensione spaziale possa usare i precedenti criteri scientifici;

- l'emergere di gerarchie soft da integrare, e in generale le integrazioni tra le diverse parti della metropoli territoriali, può avvalersi dell'elaborazione del concetto di reti territoriali, ma il fervore intorno a questa tematica sembra raffreddarsi mentre cresce una maggiore domanda di conoscenza e di elaborazione;

- la fruizione dei servizi collettivi in una situazione "diffusa" pone problemi di accessibilità e di distribuzione molto più complessi che nella situazione concentrata. E' proprio questo il settore che merita un rilevante sforzo sia concettuale che di strumenti. L'utilizzazione al meglio delle innovazioni tecnologiche sia per rompere la standardizzazione tradizionale dei servizi collettivi che la loro distribuzione nello spazio è la strada, ma non può essere tradotta in pura "pratica". In sostanza uno sforzo notevole scientifico (anche della scienza dell'organizzazione) andrà fatto per adeguare alla nuova condizione insediativa i servizi collettivi e insieme influenzare l'insediamento in base all'articolazione dei servizi.

- gli "spazi pubblici", come si è osservato, costituiscono il punto di maggior deficit in una metropoli territoriale auto-organizzata. Ma nella metropoli territoriale si impone l'esigenza di una nuova concezione dello spazio pubblico. A questo proposito si può osservare la necessità di affiancare a quelli di tipo tradizionali (si è comunque conservatori) spazi di nuova concezione per i quali si hanno poche indicazioni e poche idee. Le nuove esigenze delle popolazione della metropoli territoriale richiedono nuovi modelli di spazio pubblico. In assenza di una nuova concezione si può osservare come esplode l'uso degli spazi privati di uso pubblico (centri commerciali e simili) e come si piegano ad esigenze sociali spazi "vuoti",più che pubblici (quelli che una terminologia nota ma inadeguata chiama "non luoghi");

- le modalità di organizzazione della metropoli territoriale deve rispondere alle esigenze di una crescita economica equilibrata. Ovviamente non è solo l'organizzazione dello spazio che può determinare lo sviluppo economico, ma sicuramente un'organizzazione attenta a questa dimensione dei fenomeni può aiutare. Come la nuova organizzazione dello spazio incida sui processi economici è noto solo in parte, né si hanno adeguate riflessioni sui meccanismi che sarebbe possibile attivare.

Giungiamo ora al punto più difficile e controverso, ma anche quello più dirimente: le sfide che riguardano il governo della metropoli territoriale.

Come più volte ripetuto, la prima sfida che la nuova organizzazione spaziale pone ai problemi di governo è la sua unitarietà. Per passare da una situazione di auto-organizzazione ad una situazione "governata", nell'ottica dell'interesse generale, 
appare indispensabile una struttura di governo (e di potere) territorialmente unica, o unitaria, che dir si voglia. L'ovvietà della precedente proposizione non ha niente a che fare con la sua realizzabilità: le amministrazioni locali sono particolarmente gelose delle proprie prerogative di potere e non sono disponibili a cederle facilmente; dall'altra il potere decentrato costituisce un notevole contributo all'attivazione dei processi democratici. In questa situazione si tratta di individuare una struttura e forma di governo (diretta, di seconda istanza, ecc.) in grado di non mortificare le prerogative degli enti locali ma anche di dare effettività al governo metropolitano.

Quindi un governo unitario, decentrato, autorevole, non centralistico ma fortemente connesso; i precedenti termini qualificativi appaiono in contraddizione tra di loro, e tali sono se si assumessero ciascuno in forma estrema, ma proprio la natura della metropoli territoriale esige che questi elementi siano coniugati in modo funzionale. La struttura di governo di cui si parla, mentre è nuova, non nasce in un vuoto ma si deve misurare con un'articolazione di poteri territoriali, diversi nei diversi paesi ma sicuramente ampi e consistenti, non eliminabili, e dovrà sviluppare tutta la sua arte (arte del governo) e tutta la sua scienza (scienza del governo) per costruire, tra gli elementi contraddittori di cui si è detto, una modalità di intervento che esalti insieme unitarietà e decentramento.

Il territorio della metropoli territoriale, sebbene caratterizzato da una debole gerarchia, non si presenta omogeneo in tutte le sue parti, cosa questa che pone diverse questione di governo. La fondamentale delle quali è quella di "resistere" alle pressioni di singole zone interne che possono chiedere, di fatto, prerogative di privilegio. Lo sguardo del governo deve essere attento ad ogni parte e guidato dal principio di equità. La declinazione di questa virtù si articola in vario modo, fermo restando che livelli di diseguaglianze spaziali e sociali sono impliciti nel meccanismo capitalistico; un governo che assuma l'equità come sua guida può contribuire a ridurre tali sperequazioni. Nella metropoli territoriale l'eliminazione o almeno l'attenuazione degli squilibri spaziali garantisce il buon funzionamento di tutto il territorio ed evita l'insorgere di eccessive gerarchie.

Proprio in quest'ambito vanno elaborati meccanismi di equa distribuzione di vantaggi e svantaggi in relazioni a processi di trasformazione del territorio che possono determinare delle entrate economiche per singoli enti locali (come il caso degli oneri di urbanizzazione o assimilabili).

Quello che si registra, anche in ragione della diffusione territoriale della popolazione, è un'attenta partecipazione dei cittadini al controllo delle trasformazioni. Si tratta di un momento di partecipazione democratica e politica molto interessante, alla quale il governo metropolitano dovrebbe porre orecchio attento e, nella misura del possibile, sollecitarla. Tale partecipazione può sfociare, tuttavia, in situazioni di esaltazioni di "egoismi territoriali" che contrastano con una visione d'insieme. Contrastare, com'è doveroso da parte di un governo metropolitano, tali egoismi territoriali, non può avvenire sulla base di "autorità", ma, per quanto possibile, attraverso un confronto puntuale tra i diversi interessi parziali e generali, nella ricerca di compensazioni e nella messa in campo di strumenti di cooperazione territoriale.

Come più volte ripetuto i processi di auto-organizzazione generano effetti sia positivi che negativi; un buon governo, in sostanza deve attivare quegli strumenti che, nell'ottica della realizzazione degli obiettivi generali, siano in grado di esaltare, aiutare, sostenere quelle pratiche sociali coerenti con gli obiettivi generali che si volessero realizzare o che a questi possono essere riportati e, invece, contrastare fino a vietare quelle pratiche che appaiono in contrasto con gli obiettivi generali.

La pianificazione metropolitana, che dovrà darsi una ricca dotazione di strumenti (politiche per i diversi aspetti), deve essere concepita come per creare nuove opportunità (economiche, sociali, culturali, ecc.) anche se pone limiti a determinate pratiche sociali. È attraverso la pianificazione che si contribuisce a creare una rinnovata condizione urbana metropolitana.

Per realizzare questo obiettivo il governo metropolitano e la sua pianificazione dovrebbero essere in grado di determinare:

- una razionale, dal punto di vista complessivo, destinazione e uso dello spazio, nell'ottica della "parsimonia", dell'articolazione delle funzioni, della ricchezza delle diversità e opportunità;

- proprio il precedente obiettivo impone un'attenta considerazione sulla redistribuzione delle risorse, nell'ottica dell'equità;

- la eliminazione degli squilibri territoriali non solo 
come affermazione di un principio di equità, ma anche e soprattutto per una migliore e maggiore qualificazione della metropoli territoriale. Si tratta di un obiettivo essenziale al funzionamento della metropoli e per garantire la possibile attivazione di tutte le potenzialità insite in questa forma di organizzazione (sia sul piano sociale, culturale e stili di vita, sia sul piano economico);

- la infrastrutturazione del territorio a garanzia della massima mobilità e della distribuzione e possibilità di fruizione dei servizi alle persone;

- l'apertura verso il "resto del mondo", dal più vicino al più lontano. Non si tratta tanto di un omaggio alla "globalizzazione", ma piuttosto al riconoscimento che da sempre l'apertura al mondo esterno migliora le condizioni di partenza, in tutti i campi e non solo in quello economico. Gli indirizzi di chiusura, i localismi più o meno accentuati, costituiscono spinte depressive sul piano sociale, culturale ed economico;

L'ideologia urbana ha affermato come la libertà sia insita alla città (la città rende liberi), ma contem- poraneamente ha fatto da velo, questo era il suo scopo, agli aspetti negativi della città (anonimato, solitudine, violenza, ecc.). Non si tratta ora di costruire una ideologia della metropoli territoriale esaltandone i meriti e negando i difetti, non di questo si tratta. Meriti e difetti della città prima (ed ora) e della metropoli territoriale solo in parte sono determinati dalla dimensione spaziale, essi sono il derivato dell'organizzazione socio-economica; come si è detto una società non equa produce un territorio della stessa qualità. Da sempre, tuttavia, la condizione urbana ha costituito un modo per mitigare questa situazione, con esiti non sempre uguali nel tempo e nello spazio; tale mitigazione, infatti, non è che frutto di governo, e il governo non può essere scisso dall'espressione della forza e della qualità della partecipazione politica dei soggetti sociali. Anche se la metropoli territoriale in se stessa presenta, o almeno a me sembra che presenti, delle condizioni più favorevoli per questa opera di mitigazione e, perché no, di trasformazione, ciò non può avvenire nel vuoto politico. 